\title{
ORIGINAL
} ARTICLES

\section{PedHITSS: A Screening Tool to Detect Childhood Abuse in Clinical Settings}

\author{
Amer Shakil, MD; Philip G. Day, PhD; Jessica Chu, MPH; Sarah B. Woods, PhD; Kate Bridges
}

\begin{abstract}
BACKGROUND AND OBJECTIVES: Though child abuse is prevalent and detrimental, health care providers fail to screen for abuse at sufficient rates to detect or preempt events. Current child abuse screening tools lack brevity and usefulness in clinical settings. To validate the Pediatric Hurt-Insult-ThreatenScream-Sex (PedHITSS) screening tool, a 5-item questionnaire designed to detect and prompt provider investigation into child abuse in clinical settings, the PedHITSS was compared to the Conflict Tactics Scale: Parent-Child Version (CTSPC) screening measure.
\end{abstract}

\begin{abstract}
METHODS: Participants included 422 pediatric patients ( $\mathrm{n}=\mathbf{2 4 2}$ nonabused; $\mathrm{n}=\mathbf{1 8 0}$ abused subsample) recruited from an ambulatory care setting, a medical center at-risk referral clinic, or homeless shelter clinic. Parents were asked to complete a cross-sectional survey, including PedHITSS and CTSPC questionnaires. Concurrent validity of PedHITSS was tested with 242 participants identified as nonabused. Construct validity was assessed with 180 participants previously identified as victims of child abuse.
\end{abstract}

RESULTS: Concurrent validity between the CTSPC and PedHITSS was strong, rs=.70 $(P<.01)$. Sensitivity and specificity for correctly identifying abuse victims ( $\leq 12$ years) was optimal at a cutpoint of one or greater. There was no significant difference in sensitivity and specificity of HITSS and CTSPC in correctly identifying victims of child abuse.

CONCLUSIONS: This study indicates that PedHITSS performs as well as CTSPC in identifying and differentiating victims and nonvictims of child abuse. PedHITSS allows health care providers to confidently screen and report suspected cases of child abuse and serves as a mechanism to confirm abuse status through validated means.

(Fam Med. 2018;50(10):763-9.)

doi: 10.22454/FamMed.2018.778329

n 2016, Child Protective Services (CPS) received 4.1 million referrals for 7.4 million children experiencing abuse or neglect across the United States. ${ }^{1}$ It is estimated that $25.6 \%$ of US children experience abuse in their lifetime, ${ }^{2}$ and 2.36 deaths per every 100,000 children are attributable to abuse or neglect. ${ }^{1}$ Prevalence and risk of child abuse varies across race, ethnicity, ${ }^{3}$ and socioeconomic strata. ${ }^{4,5}$ Despite this pervasiveness, health care providers fail to screen for abuse at rates sufficient to detect or preempt events. ${ }^{6}$

Research suggests that a majority of mothers in the United States favor primary care providers asking about child abuse in clinical and emergency department settings, yet only a small percentage reported being asked about child abuse. ${ }^{7}$ Further, most injuries stemming from child abuse are likely to go undetected or unreported. ${ }^{8}$ This results, in part, from providers who experience barriers to reporting suspected abuse to protective services, anxiety specific to reporting, and lack of training or ability to differentiate abuse from nonabuse. ${ }^{9,10}$ Evidence indicates that $35 \%$ to $50 \%$ of child abuse cases recur without appropriate detection and intervention, suggesting that the inability to capture these cases can lead to a cycle of undetected child abuse. ${ }^{11}$

The lack of screening and detection of child abuse or maltreatment in health care may be attributable to the unavailability of brief screening tools developed and validated for use in clinical settings. ${ }^{12}$ Standardized screening instruments such as the 160-item Child Abuse Potential Inventory, ${ }^{13}$ the 82 -item International Society for the Prevention of Child Abuse and Neglect Child ICAST questionnaire, ${ }^{14}$ and the 22 item Conflict Tactics Scale: ParentChild Version (CTSPC) ${ }^{15}$ have been used for research purposes, but these lengthy assessments are burdensome and are neither intended nor appropriate for screening in

From the University of Texas Southwestern Medical Center, Department of Family and Community Medicine (Drs Shakil, Day, and Woods, and Ms Bridges), and Department of of Physician Assistant Studies (Ms Chu). 
outpatient settings. ${ }^{16}$ It is critical to address the dearth of brief screens for child abuse, and the limitations of extant tools in order to improve health care provider assessment, recognition, and intervention for pediatric patients experiencing child abuse.

\section{Present Study}

This study examines the psychometric properties and diagnostic accuracy of a brief screen for child abuse, the Pediatric Hurt-Insult-ThreatenScream-Sex (PedHITSS) tool. PedHITSS is completed by parents or guardians of pediatric patients $(\leq 12$ years), and includes five Likertscaled items measuring acts of commission. The purpose of the tool is to promote provider awareness of physical and sexual child abuse and to prompt an investigatory process, including the potential need to engage CPS. PedHITSS is an adaptation of the family violence HITS tool, used in adult primary care populations; this measure has been repeatedly demonstrated as valid, reliable, and feasible for use in clinical care. ${ }^{17-20}$ PedHITSS uses the four valid HITS items and was adapted by adding a question about sexual abuse. We explored the reliability, validity, sensitivity, and specificity of the PedHITSS, including its ability to detect child abuse among a sample of parents/guardians whose children have confirmed abuse experiences, in comparison to a community pediatric nonabused sample.

\section{Methods}

\section{Setting and Participants}

Data were collected over 3 years (2014-2017) from two samples of English- or Spanish-speaking parents/guardians of pediatric patients (ages 0-12 years) in the Dallas metropolitan area $(\mathrm{N}=422)$. A nonabused subsample included 242 parents/ guardians of patients seen in five ambulatory care settings for routine medical problems. An abused subsample included 180 parents/guardians of confirmed victims of child abuse either receiving treatment at a medical center's at-risk referral clinic or residing in four area shelters. Three participants in the nonabused subsample reported their child had experienced sexual abuse; these children were removed from analyses $(\mathrm{N}=419)$.

\section{Data Collection}

The University of Texas Southwestern Medical Center Institutional Review Board mandated that only verbal consent could be collected from participants; written consent was not documented due to the possibility that an abusive family member with access to the patient's medical record could discover that abuse was disclosed.

Specific to the nonabused subsample, physicians and staff confirmed participant eligibility and participants were recruited by study staff during a regular office visit. Participants were told participation was confidential and would not affect patient care. No personally identifying information was collected. Each parent/guardian was provided two measures: PedHITSS and CTSPC, for paper-and-pencil self-administration. The sequencing of the questionnaires was counterbalanced to avoid presentation effects. Participants completed the assessments privately during a medical visit or, specific to the abused subsample, after a counseling appointment. Participation was voluntary; no incentive was provided.

Specific to the abused subsample, abused status was confirmed and verified by: (1) reviewing parental admission of abuse, participant medical history, and CPS reports for victims recruited at shelter sites and the referral clinic, and (2) confirming abuse using the shelter intake procedures for children residing at a shelter who did not have a CPS report on file.

\section{Instruments}

PedHITSS. PedHITSS is a modification of HITS for adults, described above $(\alpha=.80),{ }^{18}$ and is intended to be completed by parents/guardians of pediatric patients (0-12 years).
PedHITSS contains five items, including, "During the last year, how often would you estimate that an immediate family member did each of the following to a child: (1) Physically hurt him/her; (2) Insult him/her or talk down to him/her; (3) Threaten him/her with physical harm; (4) Scream or curse at him/her; or (5) Force him/her to have sex?" Participants responded to each item using a 5 -point Likert scale $(0=$ Never, $1=$ Rarely, $2=$ Sometimes, $3=$ Fairly Often, or $4=$ Frequently). Scale scores were calculated by summing responses, with scores ranging from 0 to 20 ; higher scores indicate more frequent abusive behavior.

Conflict Tactics Scale: ParentChild Version. We used the Conflict Tactics Scale: Parent-Child Version (CTSPC) to evaluate concurrent and discriminant validity. ${ }^{15,21}$ CTSPC was designed to measure childhood maltreatment using parent/guardian self-report. ${ }^{15}$ CTSPC contains 22 items and has been validated to measure child maltreatment in children from infancy to 17 years of age using the following subscales: nonviolent discipline (four items), psychological aggression (five items), and physical assault (13 items). ${ }^{15}$ We included two additional sexual abuse items that are adjunctive to the CTSPC. ${ }^{15}$ Items are prompted with, "During the last year, how often would you estimate that an immediate family member has done each of the following?" Parents/guardians then indicate how often nonviolent and violent specific scenarios (eg, "You threw or knocked down your child") occurred. Specifically, the frequency of each example of maltreatment was rated using a 7-point Likert scale ranging from 0 (never) to 6 (more than 20 times). Responses were then recoded such that responses range from 0 to $25 .^{15}$ The two sexual abuse items utilize a unique 3-point Likert scale ranging from 0 (never) to 2 (more than once). Following recoding, responses were summed within subscales..$^{15} \mathrm{~A}$ total scale score was also calculated for the full CTSPC (range 0-550), 
and the full CTSPC with the addition of the two sexual abuse items (range 0-554).

\section{Data Analysis}

Reliability. The Cronbach $\alpha$ (ie, internal reliability) of the PedHITSS and CTSPC scales were calculated for the present sample; both Cronbach $\alpha$ and specific interitem correlations were reported.

Validity. Convergent and discriminant validity was assessed using Pearson correlation to calculate within-participant concordance between PedHITSS responses and responses on corresponding CTSPC subscales. Convergent validity (ie, similarity between PedHITSS and CTSPC responses in corresponding content areas) is determined by strong, positive, significant correlations $(r>.60$, $P<.05) .{ }^{22}$ Discriminant validity (ie, dissimilarity between PedHITSS and CTSPC responses in corresponding content areas) is determined by nonsignificant, weak correlations $(r<.40, P>.05)$. Specifically, we predicted strong, significant correlations between the following PedHITSS items-CTSPC subscales: (1) hurtphysical assault; (2) insult-psychological aggression; (3) threaten-physical assault; (4) scream-psychological aggression and nonviolent discipline; and (5) sex-sexual abuse.

Lastly, construct validity of the PedHITSS is examined using factor analysis. The Kaiser-Meyer-Olkin measure of sampling adequacy, Bartlett's test of sphericity (a test of the correlation matrix, with results indicated by $\chi^{2}$ ), and shared variance across scale items $\left(h^{2}\right)$ is reported. The first two tests should be closer to 1 , and significant, respectively, to indicate a factor analysis of the individual PedHITSS items is possible. Exploratory factor analysis (EFA), using maximum likelihood estimation, is first used to examine the number of potential factors as determined by eigenvalue (percent of the variance captured). Confirmatory factor analysis (CFA) with varimax rotation is then used to specify the number of factors. Model fit for the EFA and CFA is reported using $\chi^{2}$.

Diagnostic Accuracy. The ability of the PedHITSS to detect whether a participant's child was in the nonabused (specificity) or abused subsample (sensitivity), was estimated using a receiver operating characteristic (ROC) curve. This curve was plotted against the CTSPC, and optimal cutpoints were distinguished for each measure. Comparisons of the specificity and sensitivity of each measure are described, as is the area under the ROC curve (AUC), a measure of diagnostic accuracy.

In addition, each PedHITSS item was recoded to indicate a negative $(0=$ Never $)$ or positive (all responses from Rarely to Frequently) response. The specificity and sensitivity of the scale calculated using dichotomous items was assessed using the same ROC procedure.

The goal of the PedHITSS is to identify pediatric patients who are experiencing child abuse, which is an adverse childhood experience predictive of lifelong problematic health outcomes. ${ }^{23}$ Therefore, we prioritize sensitivity of the measure (ability to detect children experiencing abuse) over specificity.

\section{Results}

Participants' ages ranged from 1871 years $($ Mean $=33.52, \mathrm{SD}=8.5$ ); the majority of parents/guardians were female and English-speaking (Table 1). Children's ages ranged from $0-12$ years (Mean=5.8, SD = 3.8). Subsequent to demographic analyses, it was discovered that three participants in the nonabused subsample reported their child had experienced sexual abuse; these children were removed from further analyses $(\mathrm{N}=419)$.

\section{Reliability}

Consistent with prior research, the CTSPC was reliable, with high internal consistency for the 22-item measure (ie, excluding the two sexual abuse items) across the full sample $(\alpha=.90)$ and nonabused $(\alpha=.77)$ and abused $(\alpha=.91)$ subsamples, as well as for the nonviolent discipline $(\alpha=.76)$, psychological aggression $(\alpha=.84)$, and physical assault $(\alpha=.88)$ subscales.

PedHITSS also demonstrated good reliability for the full sample $(\alpha=.85)$, as well as for the nonabused $(\alpha=.81$, excluding the PedHITSS item specific to sexual abuse, as no participants responded positively) and abused subsamples $(\alpha=.81)$. Interitem correlations are presented in Table 2 .

\section{Convergent and Discriminant Validity}

The CTSPC (24-item) and PedHITSS scale totals were strongly correlated $(r=.70, P<.01)$. Further, the two measures were significantly correlated within expected coordinated PedHITSS item-CTSPC subscales (Table 2), indicating convergent validity. For example, the PedHITSS scream item was positively, strongly, significantly correlated with the CTSPC psychological aggression subscale, as predicted. Disparate PedHITSS items and CTSPC subscales (eg, the PedHITSS threaten item and CTSPC sexual abuse subscale) were weakly and nonsignificantly correlated, providing evidence of discriminant validity.

\section{Construct Validity}

Results of the Kaiser-Meyer-Olkin measure of sampling adequacy (.83) and Bartlett's test of sphericity $\left(\chi^{2}=1017.03, P<.01\right)$ suggested a factor analysis was possible. The EFA determined the number of factors, specified by eigenvalue, to be one. The one-factor model accounted for $63 \%$ of the variance of the five items, with an eigenvalue of 3.15 (model 
Table 1: Characteristics of Total Sample

\begin{tabular}{|c|c|c|c|}
\hline Characteristics & $\begin{array}{l}\text { No. (\%) of Total } \\
(n=422)\end{array}$ & $\begin{array}{c}\text { No. }(\%) \text { of } \\
\text { Abused }(n=180)\end{array}$ & $\begin{array}{c}\text { No. }(\%) \text { of } \\
\text { Nonabused } \\
(n=242)\end{array}$ \\
\hline \multicolumn{4}{|l|}{ Parents/guardians } \\
\hline \multicolumn{4}{|l|}{ Language of survey administered } \\
\hline English & $338(80.1)$ & $161(89.4)$ & $177(73.1)$ \\
\hline Spanish & $84(19.9)$ & $19(10.6)$ & $65(26.9)$ \\
\hline \multicolumn{4}{|l|}{ Sex } \\
\hline Female & $380(90.0)$ & $173(96.1)$ & $207(85.5)$ \\
\hline Male & $37(8.8)$ & $5(2.8)$ & $32(13.2)$ \\
\hline \multicolumn{4}{|l|}{ Race/ethnicity } \\
\hline Hispanic & $194(46)$ & $62(34.4)$ & $132(54.5)$ \\
\hline White & $85(20.1)$ & $38(21.1)$ & $47(19.4)$ \\
\hline Black & $116(27.5)$ & $72(40)$ & $44(18.2)$ \\
\hline Asian & $18(4.3)$ & $3(1.7)$ & $15(6.2)$ \\
\hline Other/mixed & $6(1.4)$ & $4(2.2)$ & $2(0.8)$ \\
\hline \multicolumn{4}{|l|}{ Parent/guardian of child in age group } \\
\hline $0-4$ years old & $143(33.9)$ & $57(31.7)$ & $86(35.5)$ \\
\hline 5-8 years old & $155(36.7)$ & $77(42.8)$ & $78(32.2)$ \\
\hline 9-12 years old & $111(26.3)$ & $46(25.6)$ & $65(26.9)$ \\
\hline \multicolumn{4}{|l|}{ Parent/guardian relationship to child } \\
\hline Parent & $386(91.5)$ & $158(87.8)$ & $228(94.2)$ \\
\hline Legal Guardian & $8(1.9)$ & $4(2.2)$ & $4(1.7)$ \\
\hline Foster Parent & $3(0.7)$ & $1(0.6)$ & $2(.8)$ \\
\hline Grandparent & $9(2.1)$ & $4(2.2)$ & $5(2.1)$ \\
\hline Live-in boyfriend/girlfriend & $8(1.9)$ & $8(4.4)$ & $0(0.0)$ \\
\hline \multicolumn{4}{|l|}{ Children } \\
\hline \multicolumn{4}{|l|}{ Sex } \\
\hline Female & $204(48.3)$ & $94(52.2)$ & $110(45.5)$ \\
\hline Male & $214(50.7)$ & $85(47.2)$ & $129(53.3)$ \\
\hline \multicolumn{4}{|l|}{ Race/ethnicity } \\
\hline Hispanic & $197(46.7)$ & $65(36.1)$ & $132(54.5)$ \\
\hline White & $82(19.4)$ & $35(19.4)$ & $47(19.4)$ \\
\hline Black & $119(28.2)$ & $74(41.1)$ & $45(18.6)$ \\
\hline Asian & $17(4)$ & $2(1.1)$ & $15(6.2)$ \\
\hline Other/mixed & $4(.9)$ & $3(1.7)$ & $1(0.4)$ \\
\hline
\end{tabular}

fit: $\left.\chi^{2}(5)=25.78, P<.01\right)$. A two-factor model accounted for a total of $82 \%$ of the variance; the second factor had an eigenvalue (variance) of .93. The variance explained by the retained factor $\left(h^{2}\right)$, for each of the five PedHITSS scale items, is reported in Table 3 , as is each item's factor loading. While four items loaded onto the individual factor, the PedHITSS sexual abuse item did not. Given this result, and the additional variance explained by the second factor, a CFA was utilized to specify two factors within the measure.

The results of the CFA $\left(\chi^{2}=1.76\right.$, $P=.185)$ demonstrated two clusters of items: (1) insult, threaten, and scream, and (2) hurt and, to a much lesser extent, sexual abuse. The two factors appear to index items reflective of verbal abuse and physical/sexual abuse. However, the model was nonsignificant. Therefore, a one-factor solution is preferable.

\section{Specificity and Sensitivity}

The PedHITSS performed superior to the CTSPC in accurately determining participant group membership 
Table 2: Correlation Between PedHITSS Responses and CTSPC Responses

\begin{tabular}{|c|c|c|c|c|c|c|c|c|c|}
\hline & 1 & 2 & 3 & 4 & 5 & 6 & 7 & 8 & 9 \\
\hline 1. Hurt item & - & & & & & & & & \\
\hline 2. Insult item & $.66^{* * * *}$ & - & & & & & & & \\
\hline 3. Threaten item & $.68^{* * *}$ & $.75^{* * *}$ & - & & & & & & \\
\hline 4. Scream item & $.57 * * *$ & $.76^{* * * *}$ & $.72^{* * * *}$ & - & & & & & \\
\hline 5. Sex item & $.25 * * *$ & $.23 * * *$ & $.19 * * *$ & $.17^{* * * *}$ & - & & & & \\
\hline 6. CTS Nonviolent Discipline Scale & $.15^{* * *}$ & $.27 * * *$ & $.29 * * *$ & $.29 * * *$ & -.00 & - & & & \\
\hline 7. CTS Psychological Aggression Scale & $.51^{* * *}$ & $.67^{* * * *}$ & $.66^{* * *}$ & $.71^{* * * *}$ & $.10 *$ & $.49^{* * *}$ & - & & \\
\hline 8. CTS Physical Assault Scale & $.57 * * *$ & $.52^{* * * *}$ & .60 *** & $.50 * * *$ & .07 & $.36 * * *$ & $.68^{* * * *}$ & - & \\
\hline 9. CTS Sex Scale & $.10^{*}$ & $.11^{*}$ & .04 & .10 & $.60 * * *$ & .01 & .08 & .07 & - \\
\hline
\end{tabular}

$* P<.05$.

**** $P<0.001$.

Table 3: Factor Loading for the PedHITSS Tool Items

\begin{tabular}{|l|c|c|c|}
\hline \multicolumn{1}{|c|}{ Item } & EFA & \multicolumn{2}{c|}{ CFA } \\
\hline Hurt item & $\mathbf{1}$ & $\mathbf{1}$ & $\mathbf{2}$ \\
\hline Insult item & .74 & .99 & -.01 \\
\hline Threaten item & .89 & .65 & .61 \\
\hline Scream item & .87 & .69 & .51 \\
\hline Sex item & .83 & .56 & .65 \\
\hline Eigenvalues & .24 & .25 & .07 \\
\hline Percent of variance & 3.15 & 3.15 & .93 \\
\hline
\end{tabular}

Abbreviations: EFA, exploratory factor analysis; CFA, confirmatory factor analysis.

(ie, nonabused versus abused). Specifically, the PedHITSS AUC was .85 ( $\mathrm{SE}=.02, P<.01,95 \%$ CI, 0.81-0.89), while the AUC for both the 22-item and 24-item CTSPC was .74 ( $\mathrm{SE}=.03$, 95\% CI, 0.69-0.80; Figure 1).

Sensitivity and specificity findings indicate that any positive answer on the PedHITSS (a score of one, using regular or dichotomous scoring) maximizes sensitivity (the probability of correctly classifying a participant as a member of the abused subsample) while also demonstrating good specificity (the probability of correctly classifying a participant as a member of the nonabused subsample; Table 4). Therefore, the optimal PedHITSS cutpoint is one, indicating that in either scoring method, a positive answer on any item requires physician follow-up. Further, 100\% of the sample is correctly classified (ie, false positives are eliminated) at a PedHITSS score of 8.5 out of 20 .

Post-hoc Analysis. Given the rarity of positive answers to the PedHITSS sexual abuse item, and the need for every provider to follow up on any positive answer to sex abuse question, we conducted a post-hoc test of the accuracy of the PedHITSS scored without this item. The results mimicked the findings above, such that a score of 1 (using regular or dichotomous scoring) produced the greatest sensitivity. In other words, a positive answer on any of the four remaining items best detected whether a participant's child was a member of the abused subsample. The full sample was correctly classified at a score of 8.5 on the four remaining items.

\section{Discussion}

This study was conducted to assess the psychometric properties and diagnostic accuracy of the 5-item PedHITSS, a novel screening tool for detecting physical and sexual abuse in children. Results indicate that the PedHITSS is reliable in nonabused and abused pediatric samples with ages less than 12 years, and valid, aligning with the widely-used CTSPC. Further, the PedHITSS was at least as effective at discriminating between abused and nonabused child subsamples as the CTSPC, indicated by a greater AUC.

Sensitivity and specificity analyses for the PedHITSS indicate that one positive answer on any of the measure's five items requires clinician follow-up to promote the greatest likelihood of capturing potential ongoing child abuse. In other words, clinicians using the PedHITSS should follow up any positive response, and ask the parent/guardian completing the assessment to describe their answer. Further, we prioritized sensitivity in our analyses in order to maximize the likelihood of capturing a serious and potentially health-altering adverse childhood experience for pediatric patients. Additional risk may be accrued by clinicians who engaged CPS for any one positive response on PedHITSS, as this could impact the patient-provider relationship and continuity of care. Instead, our present preliminary analyses 
Figure 1: Receiver Operating Characteristics (ROC) Curves for HITSS Tool and CTSPS Measure

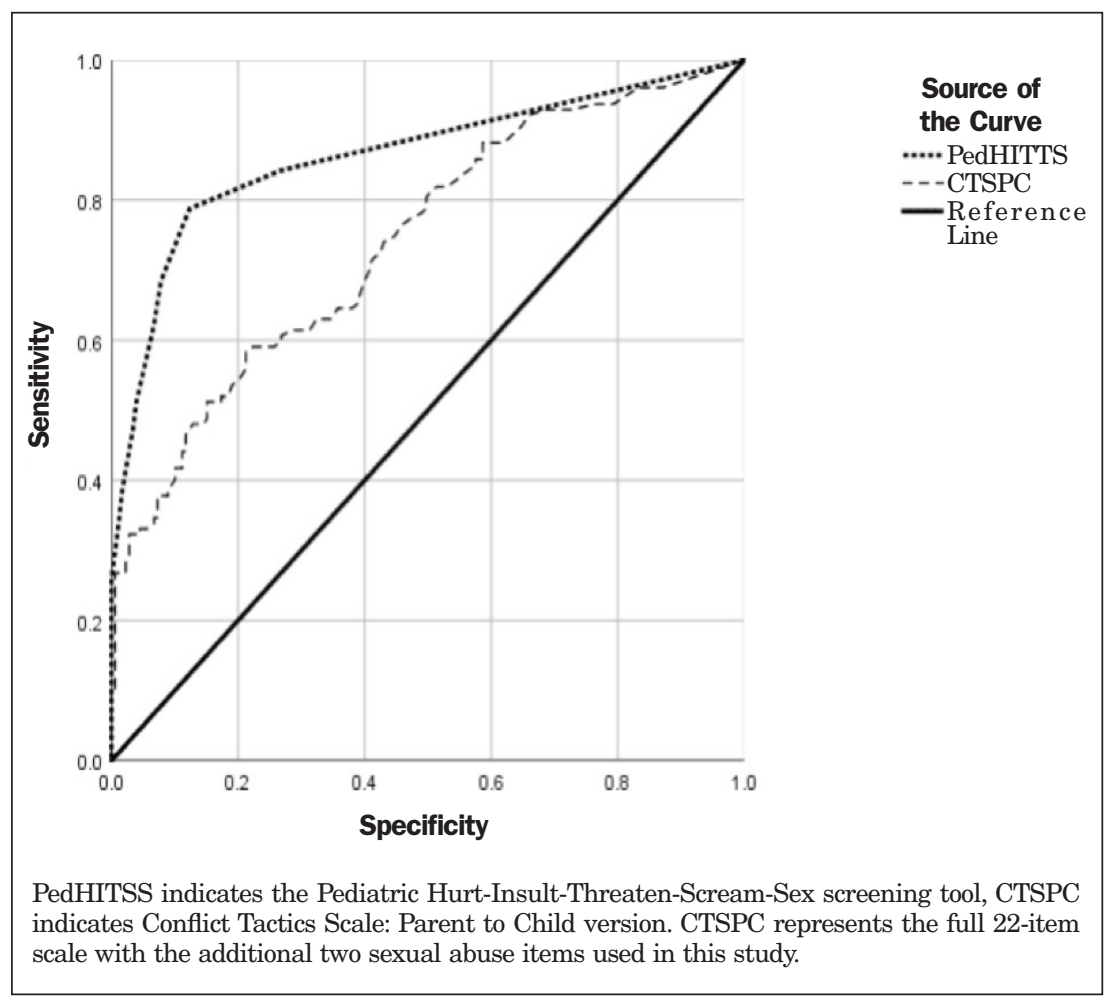

indicate that scores greater than eight may indicate that engaging protective services is beneficial, as this score correctly identified participants in the abused child subsample $100 \%$ accurately (ie, a specificity score of 1.00).

The CTSPC is an example of a child abuse measure that is lengthy and with complicated scoring methodology. Further, it does not provide established clinical cutoffs that would indicate abuse to health care providers ${ }^{15}$ rendering the measure impractical for use in clinical settings. Conversely, the PedHITSS is brief, with simple scoring, and provides a clinical cutoff score indicating the need to follow up on any positive PedHITSS response. Taken together, these findings suggest that the PedHITSS may be an effective clinical screening tool for child abuse in pediatric populations.
Limitations and Future Research The results of this study should be considered in light of its limitations. First, inherent to the administration of the measure, the PedHITSS was completed by a parent or guardian. We expect that abuse status misclassification rates were low given the extensive inclusion criteria for our abused subsample. However, this procedure may introduce misclassification depending on respondent awareness or likelihood to report abuse. Conversely, given the age range of 0-12 years, it would be impossible for the majority of children this age to complete the measure themselves. Social desirability bias can impact any sensitive survey like screening for child abuse; however, research shows that $87 \%$ of mothers wished their children's primary care physician screened for child abuse. ${ }^{7}$ To minimize the socially desirable response affect, we recommend
HITSS tool be administered in an electronic format (eg, computer or tablet given to the parent/guardian of the child while waiting in the exam room). Future research should investigate the clinical utility of this measure, including physician followup directed at the parent/guardian versus follow-up questions directed at the child patient.

Relatedly, while a strength of the present study was to offer maximum protection to participants by not requiring documentation of their identifying information, postparticipation follow-up was impossible. Future research investigating the use of the PedHITSS in clinical settings should include follow-up interviews by behavioral health providers to fully assess the extent of abuse. This could aid in the process of integrating behavioral health care, and examining the use of established psychosocial measures to flag patients and families in need of supportive care.

Finally, we compared the PedHITSS to the gold standard CTSPC. However, while the PedHITSS measures abusive acts committed against children, it does not assess neglect nor, conversely, parental warmth and engagement. Research shows $78 \%$ of child maltreatment included acts of omission, such as child neglect. ${ }^{24}$ Future research should examine additional measures that may support or complement the utility of the PedHITSS in clinical settings.

\section{Conclusion}

The PedHITSS screening tool performed as well as the CTSPC in correctly classifying abused and nonabused children, and offers greater utility to providers by supplying an actionable cut score. The findings of this study suggest that PedHITSS is a valid and reliable tool to screen for physical and sexual abuse in children ages 12 years and under in clinical settings and can help health 
care providers detect child abuse and initiate appropriate intervention.

Although the performance of the PedHITSS under research conditions is notable, providers should investigate whenever their clinical judgment leads them to suspect child abuse, regardless of the score generated by any screening instrument. Neither the PedHITSS nor any other tool should be used in lieu of good judgment, and clinical acumen should outweigh test scores if a discrepancy between the two emerges.

ACKNOWLEDGMENTS: The authors acknowledge the University of Texas Southwestern Medical Center Department of Family and Community Medicine for continued support.

FINANCIAL SUPPORT: This study was supported by Texas Academy of Family Physicians Foundation.

CORRESPONDING AUTHOR: Address correspondence to Philip G. Day, PhD, University of Texas Southwestern Medical Center, Department of Family and Community Medicine, 5920 Forest Park Road, Suite 650, Dallas, TX 75325. 214-648-4162. Fax: 214-266-0313. Philip.Day@utsouthwestern.edu.

\section{References}

1. US Department of Health and Human Services Administration for Children and Families, Administration on Children Youth and Families, Children's Bureau. Child maltreatment 2016. Washington, DC: US Government Printing Office; 2018.

2. Finklehor D, Turner H, Shattuck A, Hamby S, Kracke K. Children's exposure to violence, crime, and abuse: an update. Rockville, MD: Office of Juvenile Justice and Delinquency Prevention, U.S. Department of Justice; 2015.

3. Maguire-Jack K, Cao Y, Yoon S. Racial disparities in child maltreatment: the role of social service availability. Child Youth Serv Rev. 2018;86:49-55.
4. Maguire-Jack K, Lanier P, Johnson-Motoyama M, Welch H, Dineen M. Geographic variation in racial disparities in child maltreatment: the influence of county poverty and population density. Child Abuse Negl. 2015;47:1-13.

5. Wildeman C, Emanuel N, Leventhal JM, Putnam-Hornstein E, Waldfogel J, Lee H. The prevalence of confirmed maltreatment among US children, 2004 to 2011. JAMA Pediatr. 2014;168(8):706-713.

6. Nygren P, Nelson HD, Klein J. Screening children for family violence: a review of the evidence for the US Preventive Services Task Force. Ann Fam Med. 2004;2(2):161-169.

7. Dubowitz H, Prescott L, Feigelman S, Lane W, Kim J. Screening for intimate partner violence in a pediatric primary care clinic. Pediatrics 2008. 2008;121(1):e85-e91.

8. Christian CW; Committee on Child Abuse and Neglect, American Academy of Pediatrics. The evaluation of suspected child physical abuse. Pediatrics. 2015;135(5):e1337-e1354.

9. Tiyyagura G, Gawel M, Koziel JR, Asnes A, Bechtel K. Barriers and facilitators to detecting child abuse and neglect in general emergency departments. Ann Emerg Med. 2015;66(5):447454.

10. Foster RH, Olson-Dorff D, Reiland HM, Budzak-Garza A. Commitment, confidence, and concerns: assessing health care professionals' child maltreatment reporting attitudes. Child Abuse Negl. 2017;67(1):54-63

11. Leetch AN, Leipsic J, Woolridge DP. Evaluation of child maltreatment in the emergency department setting: an overview for behavioral health providers. Child Adolesc Psychiatr Clin N Am. 2015;24(1):41-64.

12. Woods SB, Priest JB, Denton WH. Tell me where it hurts: assessing mental and relational health in primary care using a biopsychosocial assessment intervention. Fam J (Alex Va) 2015;23(2):109-119

13. Milner JS. The Child Abuse Potential Inventory: Manual. Webster, NC: Psytec; 1980.

14. Zolotor AJ, Runyan DK, Dunne MP, et al. ISPCAN Child Abuse Screening Tool Children's Version (ICAST-C): instrument development and multi-national pilot testing. Child Abuse Negl. 2009;33(11):833-841.
15. Straus MA, Hamby SL, Warren WL. Conflict tactic scales handbook and revised forms. Los Angeles, CA: Western Psychological Services; 2003.

16. Moyer VA, US Preventive Services Task Force. Primary care interventions to prevent child maltreatment: US Preventive Services Task Force recommendation statement. Ann Intern Med. 2013;159(4):289-295.

17. Chan CC, Chan YC, Au A, Cheung GOC. Reliability and validity of the 'Extended-Hurt, Insult, Threaten, Scream' (E-HITS) screening tool in detecting intimate partner violence in hospital emergency departments in Hong Kong. Hong Kong J Emerg Med. 2010;17(2):109-117.

18. Sherin KM, Sinacore JM, Li X-Q, Zitter RE, Shakil A. HITS: a short domestic violence screening tool for use in a family practice setting. Fam Med. 1998;30(7):508-512.

19. Shakil A, Donald S, Sinacore JM, Krepcho M. Validation of the HITS domestic violence screening tool with males. Fam Med. 2005;37(3):193-198.

20. Shakil A, Bardwell J, Sherin K, Sinacore JM, Zitter R, Kindratt TB. Development of Verbal HITS for intimate partner violence screening in family medicine. Fam Med. 2014;46(3):180185

21. Riina EM, Lippert A, Brooks-Gunn J. Residential instability, family support, and parent-child relationships among ethnically diverse urban populations. J Marriage Fam. 2016;78(4):855870

22. Evans JD. Straightforward statistics for the behavioral sciences. Pacific Grove, CA: Brooks/ Cole Publishing; 1996.

23. McKelvey LM, Conners Edge NA, Fitzgerald S, Kraleti S, Whiteside-Mansell L. Adverse childhood experiences: screening and health in children from birth to age 5. Fam Syst Health. 2017;35(4):420-429.

24. US Department of Health and Human Services AfCaF; Administration on Children, Youth and Families; Children's Bureau. Acts of omission: an overview of child neglect. Child Welfare Information Gateway. 2012 